\title{
Perfil dos recém-nascidos a termo admitidos na Unidade de Terapia Intensiva Neonatal da Maternidade Escola Assis Chateaubriand
}

\section{Profile of term newborn admitted in the Neonatal Intensive Care Unit of Maternidade Escola Assis Chateaubriand}

Ana Caroline Dantas Guedes de Moura ${ }^{1}$. Maria Francielze Holanda Lavor ${ }^{2}$. Victor de Alencar Moura ${ }^{3}$

1 Médica pediatra, residente em Neonatologia, Maternidade Escola Assis Chateaubriand (MEAC), Universidade Federal do Ceará (UFC), Fortaleza, Ceará, Brasil. 2 Mestre em Saúde Pública pela Universidade Federal do Ceará (UFC), Especialista em Pediatria, Neonatologia e Medicina Intensiva Pediátrica. Médica da Maternidade Escola Assis Chateaubriand (MEAC-UFC) e Instituto Dr. José Frota, Fortaleza, Ceará, Brasil. 3 Médico Ginecologista e Obstetra. Mestrando no Mestrado Profissional em Saúde da Mulher e da Criança, Universidade Federal do Ceará (UFC), Fortaleza, Ceará, Brasil.

\section{RESUMO}

Objetivo: avaliar a admissão de recém-nascidos a termo (RNTs) na Unidade de Terapia Intensiva Neonatal (UTIN), considerando as principais causas e o desfecho final. Métodos: trata-se de um estudo retrospectivo, observacional com análise documental de prontuários, realizado na Maternidade Escola Assis Chateaubriand, no período de janeiro a junho de 2016. Os dados foram consolidados utilizando o programa Statistical Package for the Social Science 22 (SPSS) e apresentados em forma de frequência simples, gráficos e tabelas. Resultados: observou-se uma taxa de admissão de RNTs na UTIN de 11,69\%, com permanência média de 6,3 dias. As principais patologias responsáveis pelos internamentos foram: respiratórias (35,7\%), malformações abdominais/ trato gastrointestinal (TGI) $(11,9 \%)$, icterícia $(7,1 \%)$, malformação diafragmática $(7,1 \%)$ e pós-cirúrgico $(7,1 \%)$. A comparação entre a necessidade de reanimação e o suporte respiratório utilizado mostrou que RNTs que não foram reanimados ficaram em ar ambiente, ao contrário dos reanimados que ficaram em ventilação mecânica invasiva (VMI), sendo um indicativo de que quanto mais comprometido após o nascimento, maiores as chances de necessidade de suporte respiratório. Conclusão: constatou-se que a assistência neonatal, desde a sala de parto até o desfecho final, com a aplicação de técnicas apropriadas de reanimação e suporte respiratório, fez com que a maioria dos RNTs admitidos tivesse alta $(71,4 \%)$.

Palavras-chave: Neonatologia. Nascimento a termo. Unidades de Terapia Intensiva Neonatal.

\section{ABSTRACT}

Objective: the main objective of this research was to identify, quantify, and analyze the main causes and the outcome of term newborns (TNBs) admitted in the Neonatal Intensive Care Unit (NICU). Methodology: this is a retrospective observational study, with documentary analysis of medical records of patients, made at Maternidade Escola Assis Chateaubriand, from January to June 2016. The data were consolidated using the SPSS 22 software and presented as absolute frequency, graphs and tables. Results: the TNBs admission to NICU was $11.69 \%$, with average stay of 6.3 days. The main pathologies that resulted in TNBs hospitalizations were: respiratory $(35.7 \%)$, abdominal malformations/TGI, $(11.9 \%)$, jaundice $(7.1 \%)$, diaphragmatic malformation $(7.1 \%)$, and post-surgery $(7.1 \%)$. The evaluation between the need of neonatal resuscitation and the respiratory support offered showed that the TNBs that were not resuscitated stayed in room-air, unlike the reanimated ones, that needed invasive mechanical ventilation (IMV), being an indication that the more committed the TNBs after the birth, the greater the chances of necessity for respiratory support. Conclusion: it was verified that the neonatal assistance, from birth room to outcome, using the appropriate resuscitation and respiratory support techniques, resulted in the discharge of the majority TNBs admitted in NICU (71.4\%).

Keywords: Neonatology. Term birth. Neonatal Intensive Care Unit.

Autor correspondente: Ana Caroline Dantas Guedes de Moura, Maternidade Escola Assis Chateaubriand, setor de Neonatologia, Rua Coronel Nunes de Melo, s/n, Rodolfo Teófilo, Fortaleza, Ceará. CEP: 60430-270. Telefone: +55 84 98865-2206. E-mail: caroldgmoura@hotmail.com Conflito de interesses: Não há qualquer conflito de interesses por parte de qualquer um dos autores.

Recebido em: 10 Fev 2017; Revisado em: 30 Mar 2017; Aceito em: 29 Abr 2017. 


\section{INTRODUÇÃO}

A Unidade de Terapia Intensiva Neonatal (UTIN) é destinada ao atendimento dos recém-nascidos considerados de alto risco para complicações e evolução para o óbito, sendo as patologias responsáveis pela admissão as mais variadas. ${ }^{1}$

O parto antes das 37 semanas completas de gestação é tradicionalmente definido como "pré-termo", entre 37 e 41 semanas e 6 dias como "a termo", e a partir de 42 semanas "pós-termo". Muita atenção é dada às complicações observadas em recém-nascidos (RNs) pré-termo e pós-termo, mas com relação aos recém-nascidos a termo (RNT), são encontrados poucos estudos. , $^{3,4}$

Os RNTs são suscetíveis a um amplo espectro de doenças. Observa-se que, em muitos casos, as manifestações clínicas destes transtornos são extensões dos efeitos patológicos já sofridos pelo feto. ${ }^{2}$

Tracy et al. ${ }^{5}$ realizaram estudo para avaliar a admissão de RNTs em UTINs na Austrália. Eles consideraram os registros de 1.001.249 mulheres que deram à luz durante o período de 1999 a 2002. As informações coletadas incluíam: fatores demográficos maternos, dados da gravidez, características do parto e resultados neonatais. Entre as mulheres de baixo risco, foram calculadas a probabilidade de admissão de RNTs nas UTINs levando em conta a idade gestacional entre 37 e 41 semanas, como também calcularam as chances de admissão na UTIN em associação a cesariana e ao nascimento instrumental, comparando-os com o parto vaginal não assistido com 40 semanas de gestação. Eles observaram que a taxa global de admissão à UTIN de RNTs foi de $8,9 \%$ para primíparas e $6,3 \%$ para multíparas.

A obtenção de dados demonstrativos da real qualidade de assistência materno-infantil para o planejamento de ações nos serviços de saúde deve levar em conta o conhecimento da população atendida nos serviços hospitalares, principalmente na UTIN. Especial atenção deve ser dada aos seguintes fatores: análise das condições de nascimento e óbitos de neonatos, informações biológicas da gestação e parto, o quantitativo de cesarianas, prematuridade, idade e escolaridade materna, baixo peso e asfixia ao nascer. ${ }^{6-8}$ Pesquisas indicam que há uma forte relação entre a idade gestacional em que é realizada uma cesariana eletiva e o risco de desconforto respiratório neonatal. ${ }^{9}$

De acordo com Gomes, ${ }^{10}$ a assistência materno-infantil, em nível nacional, muitas vezes tem deixado a desejar, havendo um distanciamento da realidade existente com o que é preconizado pelas políticas públicas de saúde. Como consequência, observa-se uma inadequação da assistência desde os níveis de atenção mais básicos até os mais complexos, como os prestados nas UTINs.

Nos Estados Unidos, a taxa de admissão na UTIN varia de 9 a $13 \%$ de todos os nascidos vivos (Pollack et al., 1998). Os RNTs precoces (entre 37 semanas e 38 semanas e 6 dias) também parecem ter aumento da morbidade quando comparados com RNs maiores de 39 semanas. Desse modo, a limitação dos nascimentos antes das 39 semanas constitui-se numa importante meta de saúde pública nos Estados Unidos. ${ }^{11,12}$

A Maternidade Escola Assis Chateaubriand (MEAC), serviço terciário do Estado do Ceará, possui 21 leitos de UTIN. Observa-se que a grande maioria dos pacientes internados são RNs pré-termo, não sendo muito comum a admissão de RNT. Tendo em vista a falta de análise dos dados referentes às admissões de RNT, esta pesquisa torna-se importante pela necessidade de identificar as principais variáveis e fatores de risco que levam a este internamento, como também ao seu desfecho final, buscando planejar e melhorar o atendimento a este grupo de pacientes. De acordo com o exposto, o principal objetivo deste trabalho, considerando os dados disponíveis do período de janeiro a junho de 2016, é avaliar a admissão de RNTs na UTIN, considerando as principais causas e o desfecho final.

\section{METODOLOGIA}

Estudo retrospectivo, observacional com análise documental de prontuários, realizado no período de janeiro a junho de 2016, constituindo-se em uma amostra de 42 RNTs admitidos na UTIN da MEAC, maternidade de referência em atenção materno-infantil do estado do Ceará, com 21 leitos de UTIN. Considerou-se como critério de inclusão todos os RNTs nascidos no período avaliado provenientes da sala de parto, da unidade de cuidados intermediários e do alojamento conjunto da instituição. Foram excluídos os pacientes com prontuário incompleto ou inconclusivo e os pacientes provenientes de outros setores da instituição que nasceram pré-termo e necessitaram de cuidados na UTIN após sua idade gestacional corrigida já ter atingido o termo (37 semanas).

Para a coleta de dados, utilizou-se uma ficha contendo variáveis relativas à idade materna, antecedente materno, realização de pré-natal, tipo de parto, idade gestacional, método para idade gestacional, boletim de Apgar, necessidade de reanimação na sala de parto, sexo, peso, estatura, perímetro cefálico, tempo de vida no momento da admissão na UTIN, suporte ventilatório ofertado na admissão à UTIN, patologia desencadeante da admissão à UTIN, tempo de permanência e desfecho evolutivo do RN.

Os dados foram consolidados utilizando o programa estatístico Statistical Package for the Social Science (SPSS) versão 22, e apresentados em forma de frequências absoluta e relativa, média, mediana, gráficos e tabelas. A frequência absoluta corresponde ao número de vezes que uma variável assume um determinado valor no intervalo de interesse. A frequência relativa foi determinada dividindo a frequência absoluta pelo número total de observações e a média foi calculada somando os valores de todas as observações no intervalo de interesse e dividindo pelo número total de observações. A mediana foi determinada organizando os valores em ordem crescente e, a amostra tendo um número ímpar de elementos, a mediana corresponde ao valor central. 
Quando a amostra possui um número par de elementos, a mediana corresponde ao valor médio dos dois valores centrais. Os dados foram comparados utilizando o teste do Qui-quadrado $\left(\chi^{2}\right)$ para tendência, considerando $95 \%$ como limite de significância $(\mathrm{p}<0,05)$.

O protocolo de pesquisa foi realizado seguindo os preceitos éticos que regem as pesquisas em seres humanos, de acordo com a Resolução 466/12 do Conselho Nacional de Saúde ${ }^{13}$ e com os critérios estabelecidos pela Declaração de Helsinki com suas modificações. ${ }^{14} \mathrm{O}$ trabalho foi iniciado após aprovação pelo Comitê de Ética em Pesquisa envolvendo seres humanos da MEAC, da Universidade Federal do Ceará.

\section{RESULTADOS E DISCUSSÃO}

Na MEAC, serviço terciário de referência do Ceará, de acordo com o relatório de gestão de 2016, foram realizados 3030 partos de janeiro a julho de 2016, com 3041 RNs nascidos vivos. Deste montante, 359 necessitaram de atendimento na UTIN, correspondendo a $11,80 \%$. Considerando apenas os RNTs, observou-se um total de 42 pacientes, ou seja, 1,38\% do total de nascimentos e $11,69 \%$ de RNs admitidos na UTIN. O fluxograma mostrado na Figura 1 ilustra detalhadamente estes dados. Spain et al. ${ }^{15}$ relata que aproximadamente 5 a $18 \%$ dos RNTs são admitidos na UTIN e que, além do uso de recursos limitados e os encargos financeiros para o sistema de saúde, essas admissões são imprevisíveis.

Figura 1. Fluxograma contendo as etapas da pesquisa e as patologias que levaram a admissão do RNT na UTIN da MEAC.

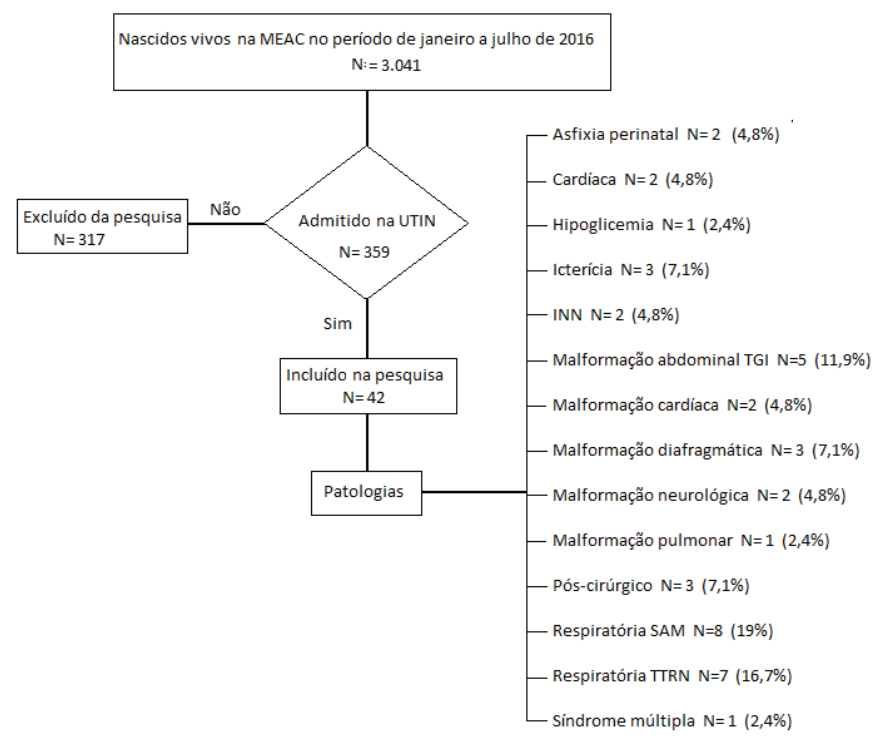

Dos 42 pacientes avaliados no trabalho, detectou-se que a idade materna média era de 26,8 anos (de 17 a 42 anos). Deste total, apenas uma não realizou pré-natal $(2,4 \%)$. Das que realizaram, observou-se um número médio de 7 consultas, o que demonstra que foi realizado o número mínimo de consultas preconizado pelo Ministério da Saúde, que determina que sejam realizadas, no mínimo, seis consultas. ${ }^{16}$
Constatou-se que 33 RNs nasceram por parto cesárea (78,6\%). As indicações para este tipo de parto foram diversas, conforme a Tabela 1, levando em consideração que a MEAC é um serviço terciário de referência materno infantil no Estado do Ceará.

Tabela 1. Indicações das cesáreas dos RNTs admitidos na UTIN da MEAC.

\begin{tabular}{lcc}
\hline Indicação cesárea & $\mathrm{N}$ & $\%$ \\
Atresia de esôfago & 1 & $3,0 \%$ \\
Cardiopatia fetal & 2 & $6,1 \%$ \\
Cesárea anterior & 5 & $15,2 \%$ \\
DHEG & 5 & $15,2 \%$ \\
DPP & 2 & $6,1 \%$ \\
Hérnia diafragmática & 3 & $9,1 \%$ \\
Malformações múltiplas & 2 & $6,1 \%$ \\
Microcefalia & 1 & $3,0 \%$ \\
Oligoamnio & 2 & $6,1 \%$ \\
Onfalocele & 2 & $6,1 \%$ \\
Placenta prévia & 1 & $3,0 \%$ \\
Síndrome & 1 & $3,0 \%$ \\
Sofrimento fetal & 5 & $15,2 \%$ \\
Teratoma & 1 & $3,0 \%$ \\
Total & 33 & $100,0 \%$ \\
\hline
\end{tabular}

*DPP: Deslocamento Prematuro da Placenta

Observou-se que $45,6 \%$ das cesarianas foram realizadas devido a cesárea anterior, doença hipertensiva específica da gestação (DHEG) e sofrimento fetal. Tendo em vista a grande preocupação com a Síndrome de Zika Congênita, foi constatada apenas uma indicação de cesárea por microcefalia ocasionada pela suspeita desta síndrome. Bolognani ${ }^{17}$ obteve resultados semelhantes avaliando dois hospitais de referência para o alto risco obstétrico e que atenderam, em 2013, a aproximadamente $20 \%$ do total de partos da Secretaria de Estado de Saúde do Distrito Federal (SES-DF, Brasília). Ela observou uma taxa de cesáreas média entre os dois hospitais de $46,5 \%$ (50,8\% e $42,3 \%)$ e que distócias, sofrimento fetal e cicatriz prévia foram as principais indicações.

Considerando a idade gestacional dos RNTs admitidos na UTIN, verificou-se uma média de 38 semanas e 5 dias, com a menor idade gestacional de 37 semanas e a maior 41 semanas e 2 dias. O Colégio Americano de Obstetras e Ginecologistas (The American College of Obstetricians and Gynecologists) e a Sociedade de Medicina Materno-Fetal (Society for MaternalFetal Medicine - SMFM) ${ }^{18}$ estão desencorajando o uso do rótulo geral de "gravidez a termo". Para estas entidades esta expressão deve ser substituída por uma série de termos mais específicos, tais como: termo precoce (entre 37 semanas completas e 38 semanas e 6 dias), termo (entre 39 semanas completas e 40 semanas e 6 dias), termo tardio (entre 41 semanas completas e 41 semanas e 6 dias). ${ }^{18}$ Foram encontrados no estudo 22 casos de termo precoce, 19 de termo e 1 de termo tardio. 
A distribuição dos métodos utilizados para identificação da idade gestacional é ilustrada no Gráfico 1, onde observa-se que os métodos mais utilizados foram ultrassonografia (USG) do primeiro trimestre e data daúltima menstruação(DUM). De acordo com Pereira et al., ${ }^{19}$ a USG do primeiro semestre é considerada o método mais preciso pelo National Institute for Health and Care Excellence e a DUM é recomendada pela Organização Mundial da Saúde (OMS) devido a elevada acessibilidade e baixo custo.

Gráfico 1. Distribuição dos métodos utilizados para identificação da idade gestacional.

\section{IG-Método}

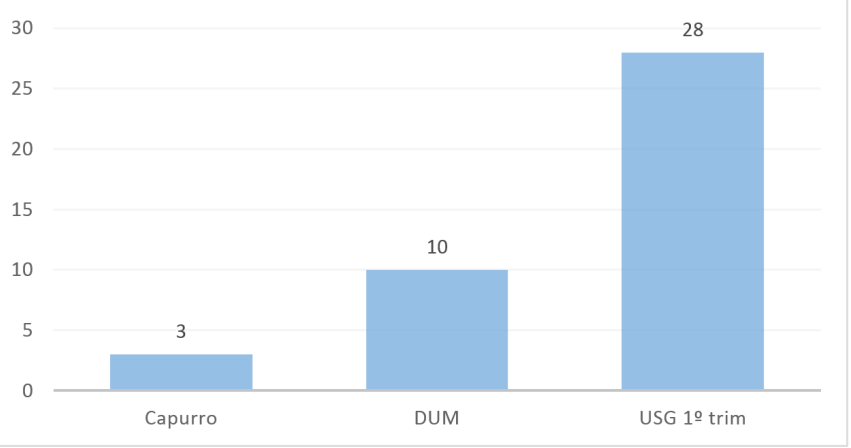

Dos RNTs admitidos, 22 eram do sexo masculino (52,4\%). O peso médio ao nascimento foi de $3,123 \mathrm{~kg}$, sendo o menor peso de $1,970 \mathrm{~kg}$ e o maior de $5,151 \mathrm{~kg}$. Observou-se que $11 \mathrm{RNTs}$ apresentaram peso ao nascimento menor que $2,5 \mathrm{~kg}(26,1 \%)$. Esses RNTs apresentaram uma alta taxa de permanência, com média de 10 dias, e 3 foram a óbito, o que demonstra a influência do baixo peso ao nascimento no desfecho final. O comprimento médio foi de $48,7 \mathrm{~cm}$, variando no intervalo de $42 \mathrm{~cm}$ a $59 \mathrm{~cm}$.

A necessidade de reanimação e seus tipos é elucidada na Tabela 2. Conforme se pode perceber, a maioria dos RNTs necessitaram de reanimação, com um total de 22 casos $(54,8 \%)$. Dentre os tipos de reanimação, o mais utilizado foi a intubação traqueal, com 13 casos $(54,2 \%)$, seguido da utilização do balão auto inflável com máscara e $\mathrm{O}_{2}$ ( 8 casos $33,3 \%$ ). A partir desses dados, observa-se que apesar da taxa de admissão de RNTs na UTIN ser baixa, isto ocorre devido à gravidade dos casos, indicada pela alta necessidade de reanimação, o que não é esperado para RNTs.

Tabela 2. Indicação de necessidade e tipos de reanimação utilizados.

\begin{tabular}{llrr}
\hline & Sim & 23 & $54,8 \%$ \\
$\begin{array}{l}\text { Necessidade de } \\
\text { reanimação }\end{array}$ & Não & 19 & $45,2 \%$ \\
& Total & 42 & $100,0 \%$ \\
\hline & Balão auto inflável sem O & 1 & $4,2 \%$ \\
& Balão auto inflável com O & 8 & $33,3 \%$ \\
Tipo Reanimação & Intubação Traqueal & 13 & $54,2 \%$ \\
& Massagem cardíaca + Drogas & 2 & $8,30 \%$ \\
& Total & 24 & $100,0 \%$ \\
\hline
\end{tabular}

Dentre as patologias responsáveis pela admissão dos RNTs na UTIN (Figura 1), as respiratórias foram responsáveis pelo maior número (35,7\%). Dentre estas, ocorreram 8 casos $(19 \%)$ de síndrome de aspiração meconial (SAM) e 7 casos (16,7\%) de taquipneia transitória do recém-nascido (TTRN). Em seguida, foi a malformação abdominal/trato gastrointestinal (TGI), com 5 casos (11,9\%). Icterícia, malformação diafragmática e pós-cirúrgico apresentaram, cada uma, 3 casos $(7,1 \%)$. Spain et al., ${ }^{15}$ considerando estudos realizados por outros autores, identificaram que os diagnósticos mais comuns para internação de RNTs em UTINs incluem: anomalias congênitas, seguidas de condições respiratórias, icterícia, hipoglicemia e infecção.

Comparando este trabalho com os resultados obtidos por Spain et al., ${ }^{15}$ observa-se uma divergência da ordem das patologias responsáveis pela admissão dos RNTs na UTIN. Nesta pesquisa, as complicações respiratórias (SAM e TTRN) predominaram. No trabalho citado, os autores observaram como causa principal as anomalias congênitas, seguida pelas complicações respiratórias. Esta divergência mostra que se deve levar em consideração a assistência obstétrica prestada ao nascimento, devido a principal patologia encontrada nesta pesquisa (SAM - 19\%) ser decorrente de sofrimento fetal. Porém, eles ressaltam que a grande maioria das crianças que nascem a termo são anatomicamente normais, e poucos estudos têm procurado identificar fatores de risco ante e intraparto para essas morbidades em RNTs.

Avaliou-se também o setor hospitalar responsável pelo encaminhamento do RNT para a UTIN, abrangendo: sala de parto $(27$ casos $-64,3 \%)$, unidade de cuidados intermediários ( 14 casos $-33,3 \%$ ) e alojamento conjunto ( 1 caso $-2,4 \%)$. O Gráfico 2 ilustra os tipos de suporte respiratório utilizados na admissão do RNT na UTIN. Verifica-se que o maior número necessitou de ventilação mecânica invasiva (VMI).

A Tabela 3 compara a necessidade de reanimação com o suporte respiratório utilizado, mostrando que RNTs que não foram reanimados ficaram em ar ambiente, sendo este tipo de suporte e o de ventilação mecânica invasiva (VMI) os únicos que apresentaram significância estatística $(p<0,05)$, ou seja, este é um indicativo de que quanto mais comprometido o RN após o nascimento maiores são as chances de necessidade de algum tipo de suporte respiratório.

Gráfico 2. Tipos de suporte respiratório utilizados na admissão do RNT na UTIN.

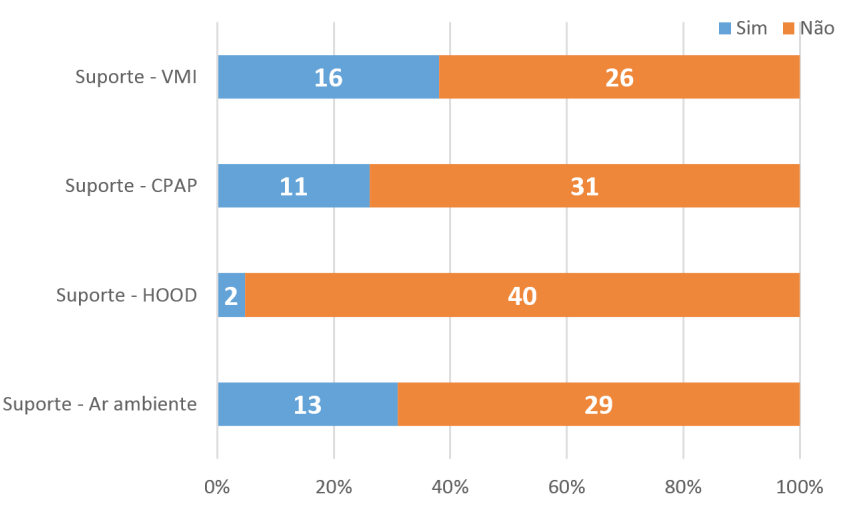


Tabela 3. Comparação entre a necessidade de reanimação e a utilização de suporte respiratório.

\begin{tabular}{lccc}
\hline & \multicolumn{3}{c}{ Necessidade de reanimação } \\
\cline { 2 - 4 } & Não & Sim & $\mathrm{N}$ \\
\hline Suporte & $\mathrm{N}(\%)$ & $\mathrm{N}(\%)$ & 0,001 \\
Ar ambiente & $11(58 \%)$ & $2(9 \%)$ & 0,111 \\
HOOD & $2(11 \%)$ & $0(0 \%)$ & 0,163 \\
CPAP & $3(16 \%)$ & $8(35 \%)$ & 0,007 \\
VMI & $3(16 \%)$ & $13(57 \%)$ & 0
\end{tabular}

*HOOD: Oxigênio em capacete; CPAP: Continuous Positive Airway Pressure.

Considerando o tempo de permanência médio na UTIN, este parâmetro variou de $1 \mathrm{~h}$ a 44 dias, com permanência média de 6,3 dias e mediana de 3 dias. Levando em consideração uma permanência superior a 10 dias, observou-se que a

\section{REFERÊNCIAS}

1. Harrison W, Goodman D. Epidemiologic trends in neonatal intensive care, 2007-2012. JAMA Pediatr. 2015;169(9):855-62.

2. Cunningham FG, Leveno KJ, Bloom SL, Spong CY, Dashe JS, Hoffman BL, et al. Williams Obstetrics. 24. ed. New York: McGraw Hill; 2014.

3. Vohr BR, Wright LL, Dusick AM, Mele L, Verter J, Steichen JJ, et al. Neurodevelopmental and functional outcomes of extremely low birth weight infants in the National Institute of Child Health and Human Development Neonatal Research Network, 1993-1994. Pediatrics. 2000;105(6):1216-26.

4. Caughey AB, Musci TJ. Complications of term pregnancies beyond 37 weeks of gestation. Obstet Gynecol. 2004;103(1): 57-62.

5. Tracy SK, Tracy MB, Sullivan E. Admission of term infants to neonatal intensive care: a population-based study. Birth. 2007;34(4):301-7.

6. Brasil. Ministério da Saúde. Secretaria de Atenção à Saúde. Departamento de ações programáticas e estratégicas. Atenção à saúde do recém-nascido: guia para os profissionais de saúde. v. 3. Brasília: Ministério da Saúde; 2011.

7. Granzotto JA, Mota DM, Real RF, Dias CM, Teixeira RF, Menta Filho JC, et al. Análise do perfil epidemiológico das internações em uma unidade de terapia intensiva neonatal. Rev Assoc Med Rio Grande Do Sul. 2012;56(4):304-7.

8. Rocha R, Oliveira C, Silva DK, Bonfim C. Mortalidade neonatal e evitabilidade: uma análise do perfil epidemiológico. Rev Enferm UERJ. 2011;19(1): 114-20.

9. Jain L, Dudell GG. Respiratory transition in infants delivered by cesarean section. Semin Perinatol. 2006;30(5): 296-304.

10. Gomes MA. Organização da assistência perinatal no Brasil. In: Moreira ME, Lopes JM, Carvalho M. O recém-nascido de alto principal patologia foi a malformação abdominal/trato gastrointestinal, que abrange uma ampla gama de patologias e necessita de cuidados especiais que são inerentes a UTIN. Por fim, avaliando o desfecho de cada caso, 30 RNTs tiveram alta $(71,4 \%), 7$ foram a óbito $(16,7 \%)$ e 5 foram transferidos para outros hospitais $(11,9 \%)$, o que demonstra a eficiência do serviço prestado na UTIN da MEAC.

\section{CONCLUSÕES}

De acordo com os resultados obtidos nesta pesquisa, fica evidente a importância da avaliação da admissão dos RNTs na UTIN da MEAC. A assistência neonatal, desde a sala de parto até o desfecho final, com a aplicação de técnicas apropriadas de reanimação e suporte respiratório, fez com que a maioria dos pacientes tivessem alta $(71,4 \%)$. A identificação das principais variáveis que levaram a este aporte, como também a avaliação do desfecho final, irá auxiliar no planejamento para melhorar o atendimento a este grupo de pacientes.

risco: teoria e prática do cuidar. Rio de Janeiro: Fiocruz; 2004. p. $21-48$.

11. Bastek JA, Sammel MD, Paré E, Srinivas SK, Posencheg MA, Elovitz MA. Adverse neonatal outcomes: examining the risks between preterm, late preterm, and term infants. Am J Obstet Gynecol. 2008;199(4):367.e1-8.

12. Cheng YW, Nicholson JM, Nakagawa S, Bruckner TA, Washington AE, Caughey AB. Perinatal outcomes in low-risk term pregnancies: do they differ by week of gestation? Am J Obstet Gynecol. 2008;199(4):370.e1-7.

13. Brasil. Conselho Nacional de Saúde. Resolução no 466, de 12 de dezembro de 2012. Brasília: Conselho Nacional de Saúde; 2012 [acesso em: 2017 Jan 20]. Disponível em: http://conselho.saude.gov. br/resolucoes/2012/Reso466.pdf

14. World Medical Association. World Medical Association Declaration of Helsinki. Ethical Principles for medical research involving human subjects. Bull World Health Organ. 2001;79(4):373-4.

15. Spain JE, Tuuli MG, Macones GA, Roehl KA, Odibo AO, Cahill AG. Risk factors for serious morbidity in term nonanomalous neonates. Am J Obstet Gynecol. 2015;212(6):799.e1-7.

16. Brasil. Ministério da Saúde. Pré-natal e puerpério: atenção qualificada e humanizada - manual Técnico. Brasília: Ministério da Saúde; 2005. Série A - normas e manuais técnicos; Série direitos Sexuais e direitos reprodutivos - caderno $\mathrm{n}^{\circ} 5$.

17. Bolognani CV. Estudo de prevalência e proposição de estratégias para a redução dos índices de cesárea na rede pública do Distrito Federal/Brasil [tese de doutorado]. Botucatu: Universidade Estadual Paulista Júlio de Mesquita Filho, Faculdade de Medicina de Botucatu; 2017. 87p.

18. ACOG - The American College of Obstetricians and 
Gynecologists; Society for Maternal-Fetal Medicine. ACOG Committee Opinion $N^{\circ}$ 579: definition of term pregnancy. Obstet Gynecol. 2013;122(5):1139-40.
19. Pereira AP, Leal MC, Gama SG, Domingues RM, Schilithz $\mathrm{AO}$, Bastos MH. Determinação da idade gestacional com base em informações do estudo Nascer no Brasil. Cad Saúde Pública. 2014;30(Sup. 1):S59-70.

\section{Como citar:}

Moura AC, Lavor MF, Moura VA. Perfil dos recém-nascidos a termo admitidos na Unidade de Terapia Intensiva Neonatal da Maternidade Escola Assis Chateaubriand. Rev Med UFC. 2018 abr-jun;58(2):19-24. 